\title{
Measuring molecular motions inside single cells with improved analysis of single-particle trajectories
}

\author{
David J. Rowland and Julie S. Biteen*
}

Department of Chemistry, University of Michigan, Ann Arbor, Michigan 48109-1055

*Email: jsbiteen@umich.edu

\section{Keywords:}

Single-molecule microscopy, super-resolution imaging, cumulative probability distribution, heterogeneous diffusion, analysis algorithms, parameter redundancy. 


\begin{abstract}
Single-molecule super-resolution imaging and tracking can measure molecular motions inside living cells on the scale of the molecules themselves. Diffusion in biological systems commonly exhibits multiple modes of motion, which can be effectively quantified by fitting the cumulative probability distribution of the squared step sizes in a two-step fitting process. Here we combine this two-step fit into a single least-squares minimization; this new method vastly reduces the total number of fitting parameters and increases the precision with which diffusion may be measured. We demonstrate this Global Fit approach on a simulated two-component system as well as on a mixture of diffusing $80 \mathrm{~nm}$ and $200 \mathrm{~nm}$ gold spheres to show improvements in fitting robustness and localization precision compared to the traditional Local Fit algorithm.
\end{abstract}




\section{Introduction}

Subcellular dynamics vary in time and over small size scales due to spatial and temporal variations such as transient interactions with molecular partners,[1] crowding by the nucleoid in bacteria,[2] or the presence of different lipid domains in membranes.[3] To measure the motions of biological molecules such as proteins and lipids in the face of this complicated local environment, single-molecule super-resolution fluorescence microscopy data must be analyzed with a method that systematically accounts for heterogeneity. One approach to single-particle tracking (SPT) derives the apparent diffusion coefficient from each individual trajectory from the mean square displacement (MSD) vs. time lag curve.[4-6] The trajectory of each particle is thus assigned an average diffusion coefficient with this MSD approach, and heterogeneous diffusion is described by dividing the collection of MSD curves into diffusive populations. The number of trajectories in each population of curves may be taken as an estimate of the relative proportions of these diffusive populations.[7-9] However, in real systems, heterogeneous diffusion can be observed even over the course of the trajectory of a single molecule, and this single-track MSD analysis specifically disallows the case where a single molecular trajectory experiences multiple diffusive modes by providing only the average diffusion coefficient for each track.

An approach that accounts explicitly for such heterogeneous motion considers the entire collection of single-molecule steps instead of dividing these steps into individual tracks. This collection of step data can then be quantified based on the cumulative probability distribution (CPD) of the collection of squared step sizes to explicitly account for spatial and temporal heterogeneities, and increase the signal-to-noise ratio. Single-step CPD analysis is therefore a diffusion estimation technique that has had impact across fields by characterizing diverse biological systems such as artificial membranes, leukocytes, bacterial membranes, neurons and artificial materials.[10-22]

Alternatively, a number of Bayesian[23-26] and machine learning algorithms[27] can be used to estimate the number of diffusive components and measure their properties, but the complexity of these methods poses a significant barrier to intuitive understanding of the underlying modes of heterogeneous motion. Fluorescence correlation spectroscopy (FCS) and the related methods of spatiotemporal image correlation spectroscopy (STICS), raster image correlation spectroscopy 
(RICS), or particle image correlation spectroscopy (PICS) can also quantify diffusion; these approaches all employ spatial or temporal correlation functions which can also be fit to multicomponent diffusion models,[28-32] but it is rare for the signal-to-noise to be high enough for the analysis of complex heterogeneous motion such as are found in bacterial systems.[33] The ability of single-particle tracking to isolate high quality trajectories from noisy single molecule data can present a more attractive conduit for analysis.

Despite its advantages, the commonly employed CPD analysis method involves a two-step fitting process where the CPD and MSD curves are fit sequentially.[11,13] We present here an approach that combines this two-step Local Fit process into a single-step multi-domain Global Fit algorithm. We analyze simulated trajectories of multiple diffusive components to measure the improvements in the diffusion coefficient estimation error and find that Global Fit is superior to the traditional Local Fit CPD analysis algorithm. We then consider the diffusion of $80 \mathrm{~nm}$ and $200 \mathrm{~nm}$ gold spheres in glycerol water solution to show that Global Fit outperforms Local Fit in a real system. We report improvements in precision, robustness, and simplicity of use.

\section{Materials and Methods}

\section{Imaging and Tracking.}

Slides were imaged at room temperature using wide-field epifluorescence microscopy in an Olympus IX71 inverted microscope with a 100×, 1.40 NA oil immersion objective (in Zeiss Immersol 518F immersion oil) and appropriate excitation, emission, and dichroic filters (Semrock LL01-488, Semrock BLP01-488 and Semrock Di01-R488, respectively). After a 3× beam expander, a Photometrics Evolve EMCCD camera with $>90 \%$ quantum efficiency captured the images at 100 frames per second. Each camera pixel corresponds to a $49 \mathrm{~nm} \times 49$ $\mathrm{nm}$ area of the sample. The gold spheres were illuminated with a $488 \mathrm{~nm}$ laser (Coherent Sapphire 488-50), that was circularly polarized with a quarter waveplate (Tower Optical AO15Z 1/4). Single molecule positions were associated into tracks with the Hungarian algorithm[34] according to an exponential merit function.[35] 
Diffusion of gold spheres in glycerol.

Gold nanoparticles with diameters 80 and $200 \mathrm{~nm}$ (BBI Solutions) were dispersed in 50\% glycerol. $5 \mu \mathrm{L}$ of the mixture was sandwiched between two glass coverslips. The second through fifth time lags were used for both the Global Fit and Local Fit algorithms to reduce the magnitude of the fitting residuals. Unweighted least squares fitting was performed with the Matlab built-in function lsqnonlin.

\section{Simulations.}

Diffusion was simulated by generating $10^{3}$ steps from a zero-mean normal distribution with variance equal to $2 D t_{\text {trame, }}$ where $D$ is the desired diffusion coefficient and $t_{\text {rame }}$ is the simulated camera exposure time which was set to 0.04 seconds. Localization precision was simulated by adding zero-mean Gaussian-distributed random numbers to the simulated trajectories; the localization precision, or the standard deviation of the random numbers, was varied from $4.9 \mathrm{~nm}$ to $73.5 \mathrm{~nm}$. Each simulation was repeated $10^{3}$ times. The first 10 time lags were used for both the Global Fit and Local Fit algorithms and unweighted least squares was performed with the Matlab built-in function lsqnonlin.

\section{Bootstrapping.}

For the analysis of the tracks of gold spheres, histograms of estimated diffusion coefficients and population weights were produced by bootstrapping the fitting procedure. The total set of 13232 squared step sizes was sampled with replacement 300 times to produce 300 unique data sets each with as many values as the original data set. These bootstrapped data sets were then fit with either the Global Fit or Local Fit method.

\section{CPD Local Fit.}

To probe heterogeneous diffusion, the cumulative probability distribution (CPD) of squared step sizes $\left(\Delta r^{2}\right)$ was calculated from the tracks of diffusing molecules at each time lag $(\tau)$ between frames in the trajectory. There is one CPD curve, $C P D_{i}$, for each time lag considered, and each 
$C P D_{i}$ was fit to the multi-term exponential fit[11] with the appropriate number of terms (three terms shown here for instance):

$$
C P D_{i}=1-\alpha_{1} \times \exp \left(\frac{-\Delta r_{i}^{2}}{M S D_{1, i}}\right)-\alpha_{2} \times \exp \left(\frac{-\Delta r_{i}^{2}}{M S D_{2, i}}\right)-\left(1-\alpha_{1}-\alpha_{2}\right) \times \exp \left(\frac{-\Delta r_{i}^{2}}{M S D_{3, i}}\right)
$$

This series of fits, where $i$, runs from 1 to the number of time lags considered, $N_{\tau}$, estimates

three mean squared displacements, $M S D_{1}, M S D_{2}$, and $M S D_{3}$, as a function of time lag for $N_{D}=3$ diffusive populations with weights $\alpha_{1}, \alpha_{2}$, and $\left(1-\alpha_{1}-\alpha_{2}\right)$, respectively. Each of the three MSD curves is then fit to a model - here of 2D unconfined diffusion - to extract the diffusion coefficient of the respective population of molecules. For example, for population 1, the secondstep fitting function is:

$$
M S D_{1}=4 D_{1} \tau+4 \sigma_{1}^{2}
$$

where $D_{1}$ is the diffusion coefficient of population $\alpha_{1}, \tau$ is the domain of time lags and $\sigma_{1}$ is the localization precision for population $\alpha_{1}$. If one uses the first 5 time lags to estimate the diffusion coefficients of three populations, the total number of fitting parameters in this local CPD fitting approach is: $5 \times N_{\tau}+2 \times N_{D}=31$ here with $N_{\tau}=5$, and $N_{D}=3$.

\section{CPD Global Fit.}

Instead of fitting in separate steps, the set of empirical CPDs may be fit all at once by incorporating the MSD functions (Equation (2)) into Equation (1). Conceptually, this can be understood as the sharing of redundant parameters, such as the weight of population 1, i.e., $\alpha_{1}$. The free parameters in the combined fitting function now include only $N_{D}$ diffusion coefficients (one for each population), a single localization precision, $\sigma$, shared among all populations, and all but one of the population weights because one is estimated using the others. For instance, if one wishes to estimate the diffusion coefficients of three populations, the total number of fitting parameters is 6 (three diffusion coefficients, one localization precision parameter, and two 
population weight parameters). This number of fitting parameters in Global Fit is hugely improved from the 31 parameters necessary for Local Fit.

We implemented Global Fit with a Matlab-specific formulation that exchanges several nonlinear least squares problems for a single larger nonlinear least squares problem. See https://github.com/BiteenMatlab/SingleMoleculeDataAnalysis for complete code; the key snippets of our code describing the use of this algorithm for a three-population Global Fit are given here:

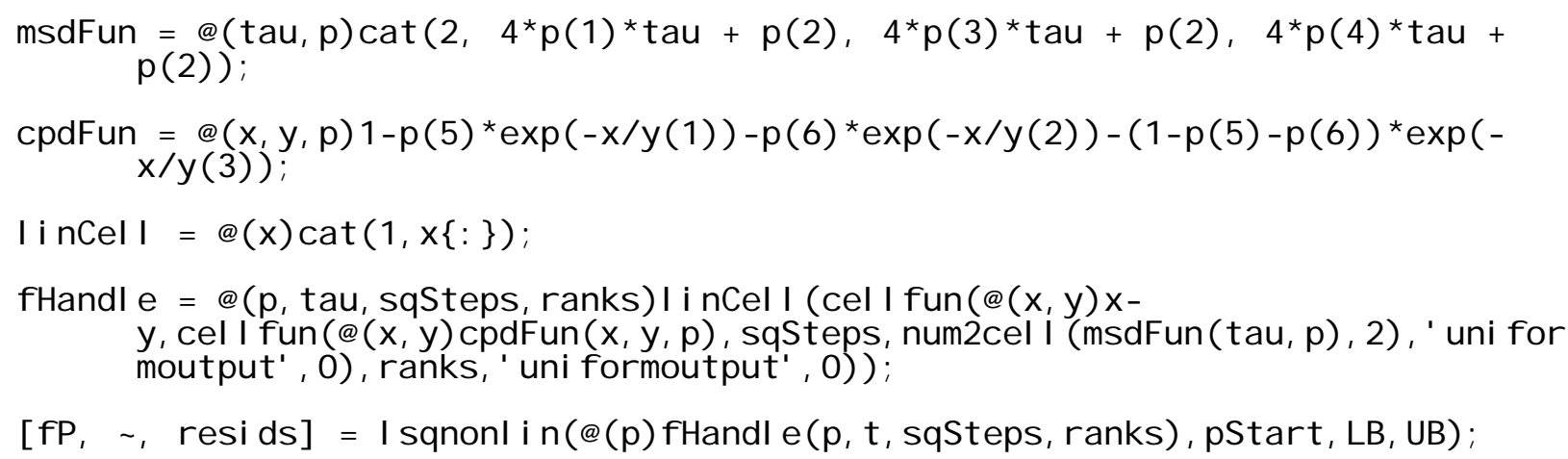

In the above code, $p$ Start is the vector of starting parameters $(1 \times 6$ in this case of three unconfined diffusive components). sqSteps is a $1 \times N_{\tau}$ cell array of the sorted lists of squared step sizes for each time lag, and ranks is a $1 \times N_{\tau}$ cell array with each element containing values from 0 to 1 having the same size as the corresponding element from the sqSteps cell array. $L B$ and $U B$ are the lower and upper bounds for the parameters found in pStart.

\section{Results and Discussion.}

To illustrate the power of CPD Global Fit, we implemented the algorithm in Matlab (Methods) and applied this analysis to simulated diffusive 2D trajectories (Figure 1A) with a combination of diffusion coefficients $\left(D_{1}=1 \mu \mathrm{m}^{2} \mathrm{~s}^{-1}\right.$ and $\left.D_{2}=0.01 \mu \mathrm{m}^{2} \mathrm{~s}^{-1}\right)$. For each trajectory, the collection of squared displacements given a certain time lag are used to compute the empirical cumulative probability distribution for that time lag. Figure 1B compares the Global Fit method to the traditional Local Fit method. In both methods, the CPD of squared step sizes is computed at each of the first 10 time lags (black and grey curves). The Local Fit method (green arrows) first fits 
each of the CPD curves independently to estimate the mean squared displacements (MSDs) of the two diffusive populations for each curve as well as to provide an average estimate of the population weights, $\alpha_{1}$ and $\left(1-\alpha_{1}\right)$. The two resulting MSD vs. time lag curves (blue and orange) are then fit in a second step to estimate the diffusion coefficients of each of the two populations. Global Fit, on the other hand, combines all of these fitting operations into a single least squares minimization step (blue arrow). This Global Fit process greatly reduces the total number of fitting parameters. In Figure 1B, Local Fit requires 3 free parameters $\left(\mathrm{MSD}_{1, \mathrm{i}}, \mathrm{MSD}_{2, \mathrm{i}}\right.$ and $\left.\alpha_{1, \mathrm{i}}\right)$ for each CPD curve fit and 2 for each MSD curve ( $D$ and the localization precision, $\sigma$ ) for a total of 34 parameters. Global Fit, which does not separate the CPD and MSD fits, in this case uses only 4 fit parameters $\left(D_{1}, D_{2}, \alpha_{1}\right.$, and $\left.\sigma\right)$.

Based on the analysis of simulations of unconfined 2D diffusion of two diffusive populations as in Figure 1A, Figure 2 describes the expected behavior of the Global Fit and Local Fit methods. At each condition, $10^{4}$ trajectories of $10^{3}$ steps were randomly generated with localization precision, $\sigma$ (Methods). Figure 2A shows the standard deviation (S.D.) of the diffusion coefficient estimates for trajectories simulated with $\sigma$ that varies from $4.9 \mathrm{~nm}$ to $73.5 \mathrm{~nm}$. At all values of $\sigma$, Global Fit (solid lines) outperforms Local Fit (dotted lines) for both diffusive components (blue and orange). Notably, once $\sigma$ approaches $70 \mathrm{~nm}$ (black arrow in Figure 2A), Local Fit completely fails to estimate the diffusion coefficient of the faster diffusive population. This breakdown is caused when numerical instability due to poor data quality produces mostly meaningless diffusion coefficient estimates. The increased numerical stability of the Global Fit algorithm enables a precise and unbiased estimation of the diffusion coefficients even in the presence of 4 diffusive populations; the Local Fit algorithm fails completely to measure motion in this situation (Supporting Information Figure S1). Overall, by constraining all CPD curves to the same diffusion model parameters, Global Fit is more robust to increased complexity and decreased data quality. The histograms in Figures $2 \mathrm{~B}$ and $2 \mathrm{C}$ present the results of the simulations used in Figure 2A at $\sigma=4.9 \mathrm{~nm}$ and $38 \mathrm{~nm}$. For this two-population simulation, at both low and high levels of localization precision, the diffusion coefficients of both populations are measured without bias by both fitting methods. However, the decreased standard deviations for diffusion coefficient estimations indicate that the results from Global Fit (solid curves) are 
more rigidly centered on their mean value. In other words, sampling error is less likely to skew the result of Global Fit when a two-term CPD model was used.

The Global Fit algorithm also shows improved performance in applications to experimental measurements where the ground truth is not known. We imaged suspensions of spherical gold nanoparticles of diameter $80 \mathrm{~nm}$ or $200 \mathrm{~nm}$ dispersed in 50\% glycerol (Methods) and tracked the particles as shown in Figure 3ABC. Figure 3D reports the bootstrapped results of Global Fit and Local Fit of a one-population diffusion model to movies of samples containing only $200 \mathrm{~nm}$ gold spheres. Global Fit results in a histogram of diffusion coefficient estimates with mean $0.24 \mu \mathrm{m}^{2} \mathrm{~s}^{-}$ ${ }^{1}$ and standard deviation $0.0061 \mu \mathrm{m}^{2} \mathrm{~s}^{-1}$, whereas local fitting of the same data set estimates a larger diffusion coefficient of $0.27 \mu \mathrm{m}^{2} \mathrm{~s}^{-1}$ and a smaller standard deviation of $0.0055 \mu \mathrm{m}^{2} \mathrm{~s}^{-1}$. Figure $3 \mathrm{E}$ shows the estimated diffusion coefficients of $80 \mathrm{~nm}$ gold spheres in $50 \%$ glycerol. Again, the mean diffusion coefficient estimate increases and standard deviation decreases when Local Fit is used instead of Global Fit. Here, the diffusion coefficient increases from $0.74 \mu \mathrm{m}^{2} \mathrm{~s}^{-1}$ to $0.82 \mu \mathrm{m}^{2} \mathrm{~s}^{-1}$ and the standard deviation decreases from $0.026 \mu \mathrm{m}^{2} \mathrm{~s}^{-1}$ to $0.022 \mu \mathrm{m}^{2} \mathrm{~s}^{-1}$ when comparing the Global Fit and Local Fit methods, respectively. See Supporting Information Table $\mathrm{S} 2$ for these results in table form. In this case of single-component diffusion, we found that Local Fit resulted in a more precise measurement of the diffusion coefficient. Importantly, this increased precision is likely due to the smaller fitting residuals that arise from having an increased number of fitting parameters in Local Fit; the increased precision should not be taken to imply an increase in accuracy, because there are no guarantees that the minima found in least squares minimization is the global minimum.

Single-particle tracks within mixtures of both nanoparticle sizes require a two-population diffusion model. Here, Global Fit more precisely estimates the diffusion coefficients. Figure $3 \mathrm{~F}$ shows histograms of the diffusion coefficient estimates for this scenario, where Global Fit estimates the two diffusion coefficients to be $0.33 \mu \mathrm{m}^{2} \mathrm{~s}^{-1} \pm 0.0074 \mu \mathrm{m}^{2} \mathrm{~s}^{-1}$ and $0.76 \mu \mathrm{m}^{2} \mathrm{~s}^{-1} \pm$ $0.047 \mu^{2} \mathrm{~s}^{-1}$ corresponding to the $200 \mathrm{~nm}$ and $80 \mathrm{~nm}$ gold spheres, respectively. Local Fit, on the other hand, estimates the diffusion coefficients to be $0.37 \mu \mathrm{m}^{2} \mathrm{~s}^{-1} \pm 0.021 \mu \mathrm{m}^{2} \mathrm{~s}^{-1}$ and 0.98 $\mu \mathrm{m}^{2} \mathrm{~s}^{-1} \pm 0.15 \mu \mathrm{m}^{2} \mathrm{~s}^{-1}$. See Supporting Information Table S2 for these results in table form. In this scenario where multiple diffusive populations are present, the improved parameter rigidity of Global Fit is readily apparent. As the complexity of the model increases, Global Fit offers a more 
robust method for estimating diffusion coefficients. It should be noted, however, that both Global Fit and Local Fit algorithms performed equally well in the estimation of the population weights, $\alpha$ and $(1-\alpha)$ (Supporting Information Figure S2).

Alternatively, we investigated the diffusion of single DiI fluorescent molecules in supported lipid bilayers (methods in Supporting Information Figure S3), and uncovered sample inhomogeneities that are outside of the scope of this work. Supporting Information Figure S4 compares the Local Fit and Global Fit methods with one and two-term fits to DiI trajectories and demonstrates the presence of two diffusive populations. DiI molecules in a homogeneous lipid environment should diffuse at around $2 \mu \mathrm{m}^{2} \mathrm{~s}^{-1}$, and indeed, the faster population has $D_{1}=2.5 \mu \mathrm{m}^{2} \mathrm{~s}^{-1}$, which is near to this value (Supporting Information Figure S5). However, the analysis also reveals a slower diffusive population $\left(D_{2}=0.4 \mu \mathrm{m}^{2} \mathrm{~s}^{-1}\right.$; Supporting Information Table $\left.\mathrm{S} 1\right)$, which is expected due to interactions between molecules of DiI and the glass surface and defects in the lipid bilayer. Global Fit outperformed Local Fit in terms of diffusion coefficient estimation precision and observe that the freely diffusing DiI accounts for $50 \%$ of the diffusing molecules as shown in Supporting Information Figure S5.

\section{Conclusions.}

In this work, we introduced global CPD fitting, a straightforward method to enhance the precision and simplicity of a commonly used diffusion coefficient estimation technique by combining redundant parameters. Instead of estimating the mean squared displacements of diffusing particles only to then fit those results in a second curve fitting step (Figure 1B), we presented a multi-domain fit that accomplishes both steps at once. The resulting reduction in degrees of freedom of the fitting operation increases the estimation precision and robustness by increasing the rigidity of the fitting parameters. The enhanced precision and robustness of the Global Fit algorithm introduced here enables precise, quantitative investigations of systems with increased complexity, for instance motion that includes more than one or two diffusive components. This Global Fit method is not restricted to freely diffusing molecules, and can be extended to any type of diffusion such as confined diffusion, diffusion with flow, or anomalous diffusion. 


\section{Artwork and Tables with Captions:}

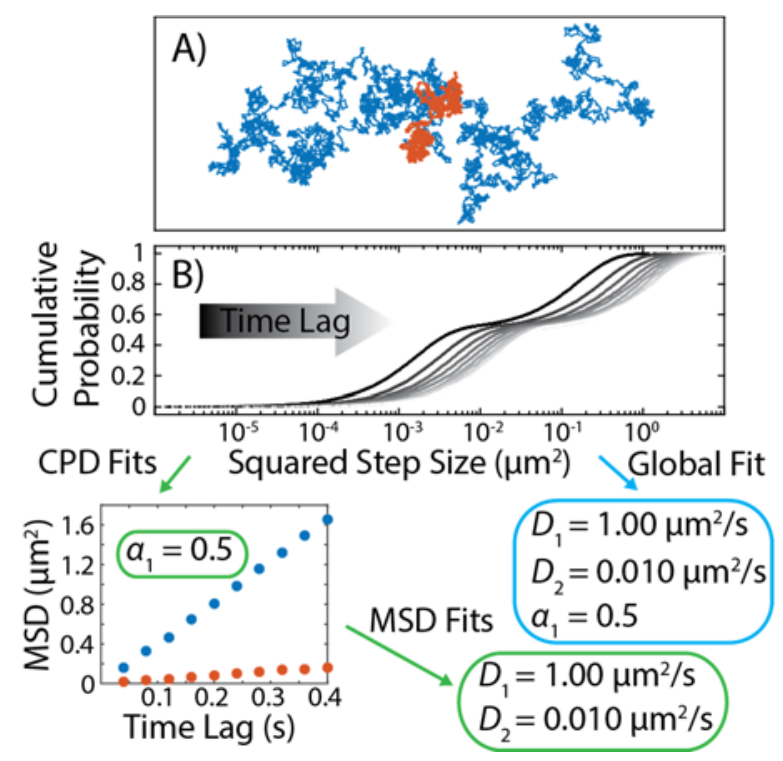

Figure 1. Schematic diagram of Local Fit and Global Fit. (A) An example pair of diffusive 2D trajectories with $10^{3}$ steps each and diffusion coefficients of $1 \mu^{2} \mathrm{~s}^{-1}$ and $0.01 \mu^{2} \mathrm{~s}^{-1}$ (blue and orange trajectories, respectively). The cumulative probability distribution (CPD) approach analyzes data from trajectories like these to extract the diffusion coefficients, $D_{1}$ and $D_{2}$, and relative weights, $\alpha_{1}$ and $\alpha_{2}=$ $\left(1-\alpha_{1}\right)$, of each diffusive population. (B) The CPD of squared step sizes is computed at each of the first 10 time lags. The resulting curves are plotted here with decreasing intensity from the first time lag (black) to the tenth (light gray). For Local Fit (green arrows), first each CPD is fit to a two-population model yielding two series of mean squared displacement (MSD) values and a population weighting factor, $\alpha_{1}$, that is the average from all the fits. The color of each resulting MSD vs. time lag curve (bottom left) indicates the color of the corresponding trajectory in (A). Next, the two MSD curves are fit separately to yield $D_{1}$ and $D_{2}$. Instead, for Global Fit (blue arrow), all $10 \mathrm{CPD}$ curves are fit at once to yield $\alpha_{1}, D_{1}$ and $D_{2}$. Local Fit here requires a total of 34 fitting parameters whereas Global Fit requires only 4 fitting parameters (two diffusion coefficients, a population weight, and a localization precision). 

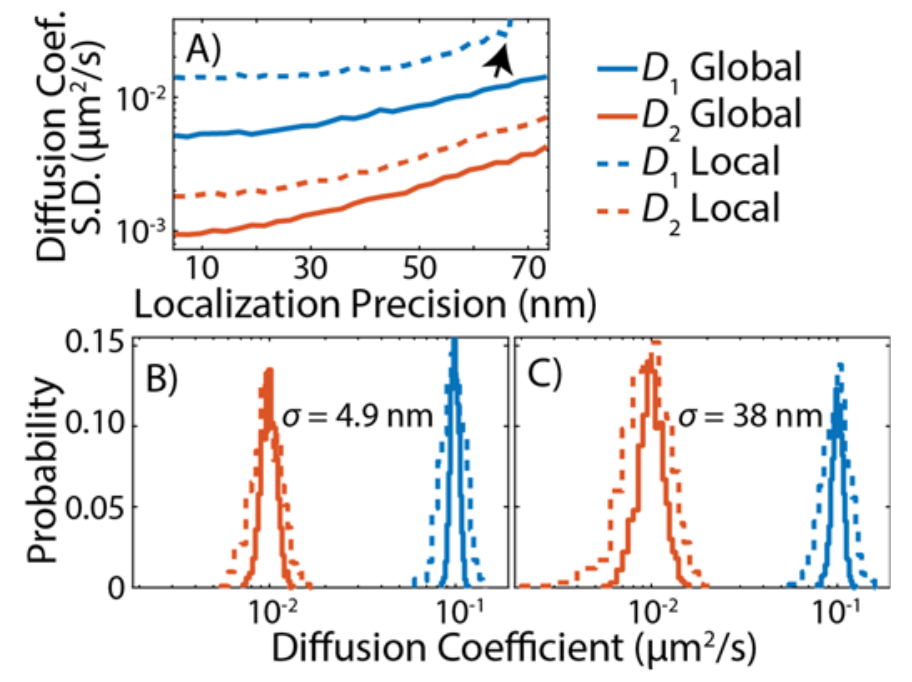

Figure 2. (A) Standard deviation of diffusion coefficient estimations for simulations of unconfined 2D diffusion of two diffusive populations. Trajectories were simulated as in Figure 1A with $10^{3}$ time steps for each of two diffusion coefficients, $0.1 \mu \mathrm{m}^{2} \mathrm{~s}^{-1}$ and $0.01 \mu \mathrm{m}^{2} \mathrm{~s}^{-1}$, and white noise was added to simulate a range of localization precisions. The trajectories of both diffusive populations at each noise level were combined then analyzed with the CPD Global Fit (blue) and Local Fit (orange) methods using a twopopulation model (Equation 1 with only the first two terms). $10^{4}$ trajectories were randomly generated and each was analyzed; the standard deviations of the estimated diffusion coefficients, $D_{1}$ and $D_{2}$, are shown. The black arrow corresponds to the point at which numerical instability due to poor data quality causes the Local Fit algorithm to fail to estimate the faster of the two diffusion coefficients. (B) and (C) Histograms of the estimated diffusion coefficients in (A) at localization precisions of $4.9 \mathrm{~nm}$ and $38 \mathrm{~nm}$, respectively. 

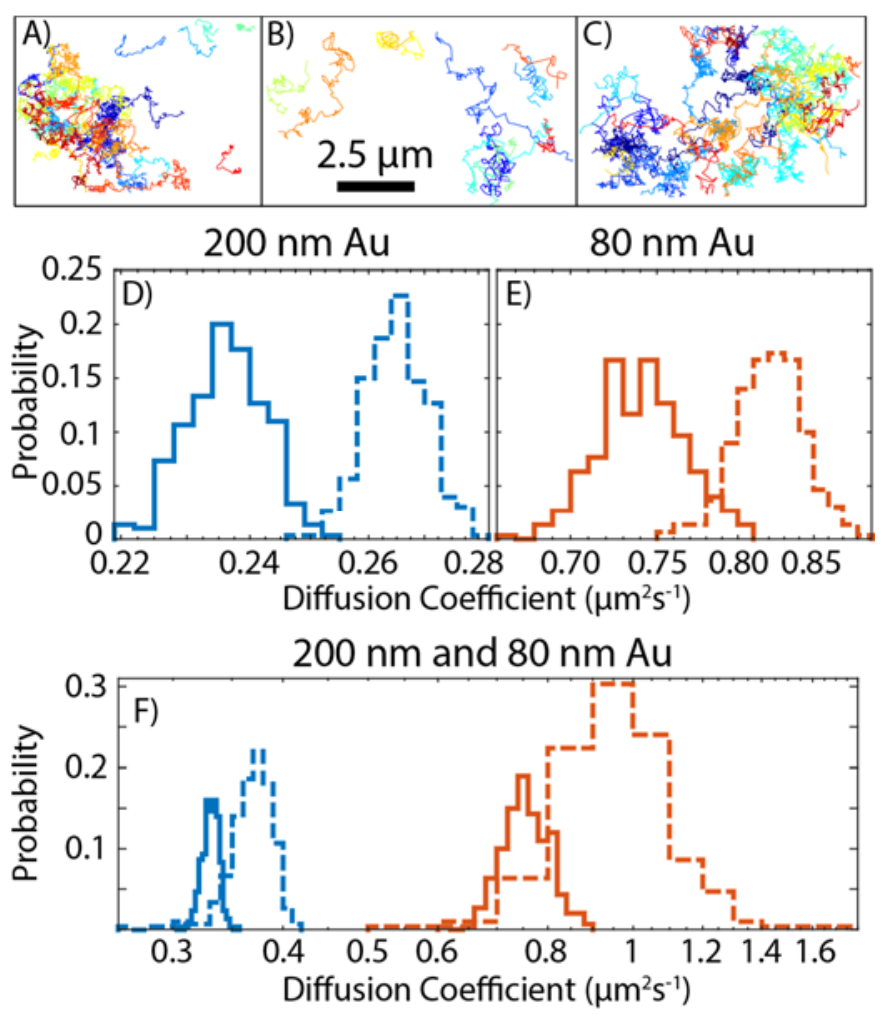

Figure 3. (A), (B), (C) 40 tracks of $200 \mathrm{~nm}$ gold spheres, 13 tracks of $80 \mathrm{~nm}$ gold spheres, and 28 tracks of a mixture of both sizes of gold spheres, all diffusing in 50\% glycerol, respectively. (D) Histograms of the estimated diffusion coefficient for $200 \mathrm{~nm}$ gold spheres in 50\% glycerol in water solution from fits to a one-component model with the Global Fit (solid line) and Local Fit (dashed line) methods. (E)

Histograms of the estimated diffusion coefficient for $80 \mathrm{~nm}$ gold spheres under the same conditions. (F) Estimated diffusion coefficients of a mixture of $200 \mathrm{~nm}$ and $80 \mathrm{~nm}$ gold spheres in a $50 \%$ glycerol in water solution fit using a two-component model by Global Fit (solid lines) and Local Fit (dashed lines). The blue and orange line colors correspond to the sphere sizes in panels ' $\mathrm{D}$ ' and ' $\mathrm{E}$ ', respectively. 


\section{Author Contributions}

DR and JB designed the research. DR developed the Global Fit algorithm, performed the simulations, carried out the experimental measurements, and analyzed the data. DR and JB discussed all the results. DR wrote the paper, which was edited by both authors. Both authors read and approved the final manuscript.

\section{Acknowledgements}

This work was funded by a National Science Foundation CAREER award (grant CHE-1252322) to JSB. Joshua Jasensky, Jason Karslake, and Joshua Karslake assisted with the lipid bilayer sample preparation, and Matthew Stone provided valuable theoretical advice. 


\section{References}

[1] B.L. Haas, J.S. Matson, V.J. Dirita, J.S. Biteen, Single-molecule tracking in live Vibrio cholerae reveals that ToxR recruits the membrane-bound virulence regulator TcpP to the toxT promoter, Mol. Microbiol. 96 (2015) 4-13. doi:10.1111/mmi.12834.

[2] S. Bakshi, B.P. Bratton, J.C. Weisshaar, Subdiffraction-limit study of Kaede diffusion and spatial distribution in live Escherichia coli, Biophys. J. 101 (2011) 2535-2544. doi:10.1016/j.bpj.2011.10.013.

[3] S.L. Veatch, B.B. Machta, S.A. Shelby, E.N. Chiang, D.A. Holowka, B.A. Baird, Correlation functions quantify super-resolution images and estimate apparent clustering due to over-counting, PLoS One. 7 (2012) e31457. doi:10.1371/journal.pone.0031457.

[4] H. Qian, M.P. Sheetz, E.L. Elson, Single particle tracking. Analysis of diffusion and flow in two-dimensional systems., Biophys. J. 60 (1991) 910-21. doi: 10.1016/S00063495(91)82125-7.

[5] A.D. Douglass, R.D. Vale, Single-Molecule Microscopy Reveals Plasma Membrane Microdomains Created by Protein-Protein Networks that Exclude or Trap Signaling Molecules in T Cells, Cell. 121 (2010) 937-950. doi:10.1016/j.cell.2005.04.009.SingleMolecule.

[6] a Kusumi, Y. Sako, M. Yamamoto, Confined lateral diffusion of membrane receptors as studied by single particle tracking (nanovid microscopy). Effects of calcium-induced differentiation in cultured epithelial cells., Biophys. J. 65 (1993) 2021-40. doi:10.1016/S0006-3495(93)81253-0.

[7] O. Rossier, V. Octeau, J.-B. Sibarita, C. Leduc, B. Tessier, D. Nair, V. Gatterdam, O. Destaing, C. Albigès-Rizo, R. Tampé, L. Cognet, D. Choquet, B. Lounis, G. Giannone, Integrins $\beta 1$ and $\beta 3$ exhibit distinct dynamic nanoscale organizations inside focal adhesions, Nat. Cell Biol. 14 (2012) 1057-1067. doi:10.1038/ncb2588.

[8] M.J. Saxton, Single-particle tracking: the distribution of diffusion coefficients., Biophys. J. 72 (1997) 1744-53. doi:10.1016/S0006-3495(97)78820-9.

[9] F. Höfling, T. Franosch, Anomalous transport in the crowded world of biological cells, Reports Prog. Phys. 76 (2013) 46602. doi:10.1088/0034-4885/76/4/046602. 
[10] M.A. Deverall, E. Gindl, E.K. Sinner, H. Besir, J. Ruehe, M.J. Saxton, C.A. Naumann, Membrane lateral mobility obstructed by polymer-tethered lipids studied at the single molecule level., Biophys. J. 88 (2005) 1875-86. doi:10.1529/biophysj.104.050559.

[11] G.J. Schütz, H. Schindler, T. Schmidt, Single-molecule microscopy on model membranes reveals anomalous diffusion., Biophys. J. 73 (1997) 1073-1080. doi:10.1016/S00063495(97)78139-6.

[12] G.J. Bakker, C. Eich, J.A. Torreno-Pina, R. Diez-Ahedo, G. Perez-Samper, T.S. van Zanten, C.G. Figdor, A. Cambi, M.F. Garcia-Parajo, Lateral mobility of individual integrin nanoclusters orchestrates the onset for leukocyte adhesion, Proc. Natl. Acad. Sci. 109 (2012) 4869-4874. doi:10.1073/pnas.1116425109.

[13] F. Oswald, A. Varadarajan, H. Lill, E.J.G. Peterman, Y.J.M. Bollen, MreB-Dependent Organization of the E. coli Cytoplasmic Membrane Controls Membrane Protein Diffusion, Biophys. J. 110 (2016) 1139-1149. doi:10.1016/j.bpj.2016.01.010.

[14] C. Hellriegel, J. Kirstein, C. Bräuchle, V. Latour, T. Pigot, R. Olivier, S. Lacombe, R. Brown, V. Guieu, C. Payrastre, A. Izquierdo, P. Mocho, Diffusion of single streptocyanine molecules in the nanoporous network of sol-gel glasses, J. Phys. Chem. B. 108 (2004) 14699-14709. doi:10.1021/jp049412a.

[15] M.L. Renner, L. Cognet, B. Lounis, A. Triller, D. Choquet, The excitatory postsynaptic density is a size exclusion diffusion environment, Neuropharmacology. 56 (2009) 30-36. doi:10.1016/j.neuropharm.2008.07.022.

[16] P.H.M. Lommerse, G.A. Blab, L. Cognet, G.S. Harms, B.E. Snaar-Jagalska, H.P. Spaink, T. Schmidt, Single-Molecule Imaging of the H-Ras Membrane-Anchor Reveals Domains in the Cytoplasmic Leaflet of the Cell Membrane, Biophys. J. 86 (2004) 609-616. doi:10.1016/s0006-3495(04)74139-9.

[17] M. Vrljic, S.Y. Nishimura, S. Brasselet, W.E. Moerner, H.M. McConnell, Translational diffusion of individual class II MHC membrane proteins in cells., Biophys. J. 83 (2002) 2681-92. doi:10.1016/S0006-3495(02)75277-6.

[18] S. de Keijzer, A. Serge, F. van Hemert, P.H. Lommerse, G.E. Lamers, H.P. Spaink, T. Schmidt, B.E. Snaar-Jagalska, A spatially restricted increase in receptor mobility is 
involved in directional sensing during Dictyostelium discoideum chemotaxis, J. Cell Sci. 121 (2008) 1750-1757. doi:10.1242/jcs.030692.

[19] J.M. Crane, A.S. Verkman, Long-Range Nonanomalous Diffusion of Quantum DotLabeled Aquaporin-1 Water Channels in the Cell Plasma Membrane, Biophys. J. 94 (2008) 702-713. doi:10.1529/biophysj.107.115121.

[20] C.L. Hsieh, S. Spindler, J. Ehrig, V. Sandoghdar, Tracking single particles on supported lipid membranes: Multimobility diffusion and nanoscopic confinement, J. Phys. Chem. B. 118 (2014) 1545-1554. doi:10.1021/jp412203t.

[21] C. Jung, J. Kirstein, B. Platschek, T. Bein, M. Budde, I. Frank, K. Müllen, J. Michaelis, C. Bräuchle, Diffusion of oriented single molecules with switchable mobility in networks of long unidimensional nanochannels, J. Am. Chem. Soc. 130 (2008) 1638-1648. doi:10.1021/ja075927e.

[22] C.K. Johnson, G.S. Harms, Tracking and localization of calmodulin in live cells, Biochim. Biophys. Acta - Mol. Cell Res. 1863 (2016) 2017-2026.

doi:10.1016/j.bbamcr.2016.04.021.

[23] F. Persson, I. Barkefors, J. Elf, Single molecule methods with applications in living cells, Curr. Opin. Biotechnol. 24 (2013) 737-744. doi:10.1016/j.copbio.2013.03.013.

[24] R. Das, C.W. Cairo, D. Coombs, A hidden Markov model for single particle tracks quantifies dynamic interactions between LFA-1 and the actin cytoskeleton, PLoS Comput. Biol. 5 (2009). doi:10.1371/journal.pcbi.1000556.

[25] A. Robson, K. Burrage, M.C. Leake, Inferring diffusion in single live cells at the singlemolecule level., Philos. Trans. R. Soc. Lond. B. Biol. Sci. 368 (2013). doi:10.1098/rstb.2012.0029.

[26] N. Monnier, Z. Barry, H.Y. Park, K.-C. Su, Z. Katz, B.P. English, A. Dey, K. Pan, I.M. Cheeseman, R.H. Singer, M. Bathe, Inferring transient particle transport dynamics in live cells, Nat. Methods. 12 (2015) 838-840. doi:10.1038/nmeth.3483.

[27] P.K. Koo, M. Weitzman, C.R. Sabanaygam, K.L. van Golen, S.G.J. Mochrie, Extracting Diffusive States of Rho GTPase in Live Cells: Towards In Vivo Biochemistry, PLoS Comput. Biol. 11 (2015) 1-26. doi:10.1371/journal.pcbi.1004297. 
[28] B. Hebert, S. Costantino, P.W. Wiseman, Spatiotemporal image correlation spectroscopy (STICS) theory, verification, and application to protein velocity mapping in living $\mathrm{CHO}$ cells., Biophys. J. 88 (2005) 3601-3614. doi:10.1529/biophysj.104.054874.

[29] C. Di Rienzo, E. Gratton, F. Beltram, F. Cardarelli, Fast spatiotemporal correlation spectroscopy to determine protein lateral diffusion laws in live cell membranes, Proc. Natl. Acad. Sci. U. S. A. 110 (2013) 12307-12312. doi:10.1073/pnas.1222097110.

[30] D.L. Kolin, D. Ronis, P.W. Wiseman, k-Space image correlation spectroscopy: a method for accurate transport measurements independent of fluorophore photophysics., Biophys. J. 91 (2006) 3061-75. doi:10.1529/biophysj.106.082768.

[31] L. Kisley, R. Brunetti, L.J. Tauzin, B. Shuang, X. Yi, A.W. Kirkeminde, D.A. Higgins, S. Weiss, C.F. Landes, Characterization of Porous Materials by Fluorescence Correlation Spectroscopy Super-resolution Optical Fluctuation Imaging, ACS Nano. 9 (2015) 91589166. doi:10.1021/acsnano.5b03430.

[32] J. Hendrix, V. Baumgärtel, W. Schrimpf, S. Ivanchenko, M.A. Digman, E. Gratton, H.G. Kräusslich, B. Müller, D.C. Lamb, Live-cell observation of cytosolic HIV-1 assembly onset reveals RNA-interacting Gag oligomers, J. Cell Biol. 210 (2015) 629-646. doi:10.1083/jcb.201504006.

[33] D.J. Rowland, H.H. Tuson, J.S. Biteen, Resolving Fast, Confined Diffusion in Bacteria with Image Correlation Spectroscopy, Biophys. J. 110 (2016) 2241-2251. doi:10.1016/j.bpj.2016.04.023.

[34] J. Munkres, Algorithms for the Assignment and Transportation Problems, J. Soc. Ind. Appl. Math. 5 (1957) 32-38. doi:10.1137/0105003.

[35] P.R. Selvin, T. Ha, Single-Molecule Techniques, Cold Spring Harbor Laboratory Press, Cold Spring Harbor, New York, 2008. 
Measuring molecular motions inside single cells with improved analysis of single-particle trajectories

\author{
D.J. Rowland and J.S. Biteen
}

\title{
Graphical Abstract
}
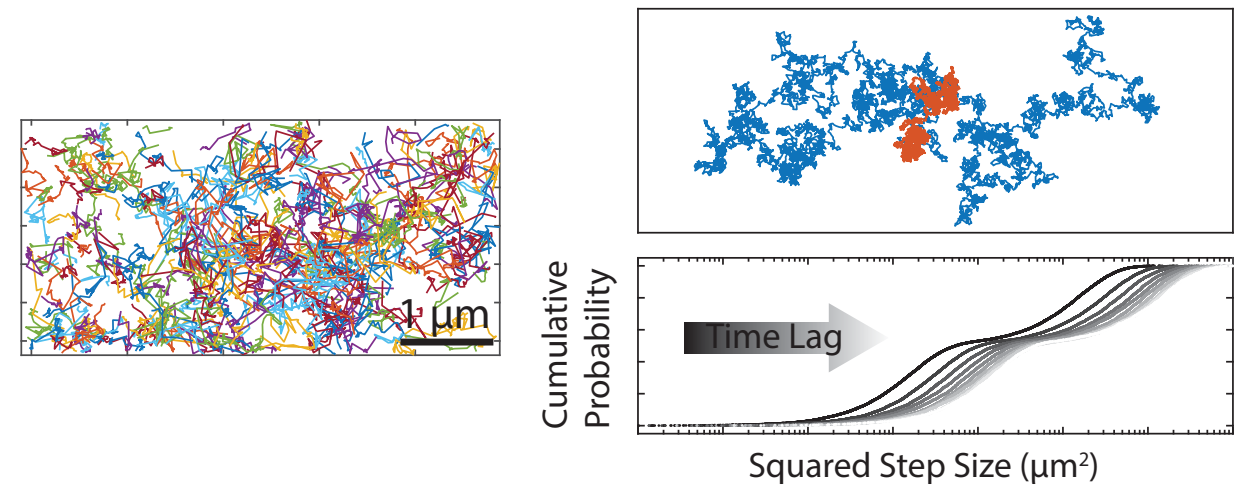